\section{The Impact of Psychological Support on Psychological Outcomes and Patients' Experiences of the Bariatric Service 1 and 2 Years after Bariatric Surgery}

Keywords: Weight loss; Psychological outcomes; Bariatric surgery; Patient satisfaction

\begin{abstract}
Background: Although bariatric surgery is widely considered the most effective form of obesity management, some patients receiving surgery describe how their psychological issues remain neglected. This study evaluated the impact of additional psychological support (a Bariatric Rehabilitation Service (BRS)) on patients' psychological outcomes at 12 months and their experiences of the bariatric service at 24 months.
\end{abstract}

Methods: Patients were randomised to receive either usual care or the BRS and rated a range of psychological outcomes at 12 months Completed psychological data was obtained from 70 patients (usua care: $n=32 ; B R S: n=38)$. At 24 months post surgery patients $(n=68)$ gave written feedback regarding the content and timing of their experiences and a sub group $(n=16)$ took part in telephone interviews about their experiences of the bariatric service they had received.

Results: By 12 months those who received the BRS reported greater improvement in their psychological outcomes compared to the usua care group in terms of vigour $\left(p=0.02\right.$, eta $\left.{ }^{2}=0.08\right)$, approach coping $\left(p=0.04\right.$, eta $\left.{ }^{2}=0.06\right)$, quality of life $\left(p=0.04\right.$, eta $\left.{ }^{2}=0.06\right)$ and self-reported hunger $\left(p=0.05\right.$, eta $\left.{ }^{2}=0.05\right)$. By 24 months a large majority of those who had received the BRS reported satisfaction with the service's timing and content. However the majority of all patients, regardless of condition, reported wanting further psychological support up to 24 months post-operatively. A minority, however, stated that as their problems were not emotional the additional support was unnec essary.

Conclusion: The BRS resulted in some small improvements in psychological outcomes. Psychological support before and after surgery could be delivered in relation to individual patient need rather than as a blanket provision. Further research is needed to detemine both the timing of such support and to identify those who would show most benefit.

\section{Introduction}

Bariatric surgery is currently the most effective intervention for weight loss for those whose BMI is greater than 40 (or 35 for those with co morbidities) [1-4]. Bariatric surgery has also been shown to result in improvements in a number of other patient outcomes including quality of life, mood, subjective health status and perceptions of eating control [4-6]. There remains significant variability in both weight change and psychological outcomes however, with a large minority of patients either not showing the desired loss of excess weight or showing weight regain by follow up [7-9]. In particular, Magro et al.

\section{Journal of Obesity and Bariatrics}

Amelia Hollywood ${ }^{1}$, Jane Ogden ${ }^{1 \star}$ and Christopher Pring $^{2}$

${ }^{1}$ Department of Psychology, University of Surrey, Guildford, Surrey GU2 7XH, UK

${ }^{2}$ Department of Laparoscopic and Bariatrics, Western Sussex Hospitals NHS Foundation Trust, St Richard's Hospital, Chichester, West Sussex PO19 6SE, UK

\section{*Address for Correspondence}

Jane Ogden, Ph.D, Department of Psychology, University of Surrey, Guildford, GU2 7XH, UK, Tel: 01483 686929; E-mail: J.Ogden@surrey.ac.uk

Copyright: (c) 2015 Hollywood A, et al.This is an open access article distributed under the Creative Commons Attribution License, which permits unrestricted use, distribution, and reproduction in any medium, provided the original work is properly cited.

Submission: 13 January 2015

Accepted: 30 April 2015

Published: 05 May 2015

Reviewed \& Approved by: Dr. Irma Corral, Assistant Professor,

Department of Psychiatry and Behavioral Medicine, East Carolina

University Brody School of Medicine, USA

found that some weight regain was observed in approximately $50 \%$ of patients (46\% within 24 months and $63.6 \%$ within 48 months) [9]. Further, patients also show variability in their changes in psychological variables after surgery [4].

To address this variability in patient outcomes some research has highlighted a role for baseline factors such as binge eating, mood or addictive behaviours as predictors of weight regain [10-13]. In contrast, other studies have focused on the mechanisms involved in failed surgery, where weight is regained or successful surgery, where weight loss is maintained, highlighting a key role for behaviour change, coping and emotional eating. For example, the results from a series of qualitative and quantitative studies indicated that whereas successful surgery is associated with a reduction in hunger and preoccupation with food and a sense of being more in control of food intake, less successful surgery is often accompanied with patients feeling unprepared for the changes required after surgery, reporting being unsupported in the time following surgery and a sense that although surgery fixes their body, psychological issues remain neglected [14-16]. This suggests that addressing these core areas may help to improve patient outcomes following surgery. It also reflects recent studies which have offered either lifestyle interventions or counselling pre or post surgery to improve outcomes $[17,18]$ and confirms the conclusions made by several research teams $[12,19,20]$ who have argued that bariatric patients require psychological input, such as assessment and behaviour change support, pre and post-surgery. Further, both National Institute for Health and Care Excellence (NICE) guidelines [21] and those by American Association of Clinical Endocrinologists (AACE) /American Association of Metabolic and Bariatric Surgery (ASMBS) /The Obesity Society (TOS) [22] state that weight loss surgery should be undertaken only by a multidisciplinary team that can provide psychological support.

In line with these suggestions the authors of the present paper carried out a randomised controlled trial to explore the impact of a health psychology led bariatric rehabilitation service (BRS) versus usual care on patient health outcomes. The bariatric rehabilitation 
Citation: Hollywood A, Ogden J, Pring C. The Impact of Psychological Support on Psychological Outcomes and Patients' Experiences of the Bariatric Service 1 and 2 Years after Bariatric Surgery. J Obes Bariatrics. 2015;2(1): 7.

service offered information, support and mentoring pre and post surgery and reflected both the rehabilitation services which are now common place for patients post heart attack and stroke [23,24], and drew upon interventions which offer patients preparation for other forms of surgery to improve post-surgical outcomes [25,26]. It also reflected a response to users of two active support groups who had highlighted the need for increased psychological input. The results of this trial showed that the intervention had no impact on change in BMI by 12 months (mean change in $\mathrm{BMI}=-16.49$ : (usual care $=$ -16.37 ; $95 \% \mathrm{CI}=15.15-17.57$; intervention $=-16.6 ; 95 \% \mathrm{CI}=15.42$ $\left.17.81 ; \eta_{\mathrm{p}}^{2}=0.001\right)$. These results have been published elsewhere [27]. The current paper presents the results relating to the impact of the intervention on psychological outcomes at 12 months after bariatric surgery. Furthermore patients' experiences of the support they received were assessed by 24 months in terms of both its timing (i.e. at what time point it should be delivered) and content, (i.e. what was and should be covered and discussed during the support sessions).

\section{Method}

\section{Sample}

Consecutive adult patients were recruited from St. Richard's Hospital in Chichester, UK, once they had been assessed by the multidisciplinary bariatric team (physician, anaesthetist, dietician, psychotherapist and surgeon) and approved for surgery. Recruitment took place over a 14-month period from October 2011 to December 2012. Those who could not effectively read or speak English were excluded as this would pose a difficulty in implementing the intervention.
One hundred and sixty two participants were recruited into the trial and weight loss data was obtained from $n=145$ by one year. Details of their demographics and the impact of the intervention on weight can be found in Ogden et al. [27]. Self reported psychological outcomes were obtained from a reduced sample of patients $(n=70$ : usual care $=32$; intervention $=38$ ) at both baseline and 1 year follow up (response rate $=43.2 \%$ ), Analysis indicated no significant differences in baseline demographics between those who completed and those who did not complete the psychological outcomes measures at one year follow up (see Table 1). Written data concerning patient experiences was obtained from patients $(n=68)$ at 24 months and qualitative data was obtained at 24 months from a sub group of patients $(n=16)$ concerning their experiences of surgery (usual care: $\mathrm{n}=8$; intervention: $\mathrm{n}=8$ ). These were sampled from those patients who had completed the 24 month follow up and consented to be contacted for a telephone interview.

\section{Design and procedures}

The study involved an open-randomised parallel group control trial with patients allocated to receive either usual care or the bariatric rehabilitation service (BRS) pre and post bariatric surgery. Details of the trial design can be found in the study protocol [28] and a previous publication [27]. Baseline psychological outcomes and weight loss were collected two weeks prior to the operation and at one year follow up. Weight was assessed in clinic and psychological outcomes were assessed via postal questionnaire. At 24 months patients were sent a short survey for both quantitative and qualitative feedback on timing and content of the intervention. In addition, a sub sample

Table 1: Patient demographics - Responders vs non responders on psychological outcomes.

\begin{tabular}{|c|c|c|c|c|}
\hline Variable & $\begin{array}{l}\text { All participants } \\
(n=183)\end{array}$ & $\begin{array}{l}\text { Psych outcomes complete } \\
\qquad(n=70)\end{array}$ & $\begin{array}{l}\text { Psych outcomes incomplete } \\
\qquad(n=113)\end{array}$ & $T / x^{2}$ \\
\hline Age (yrs) & $\begin{array}{c}\text { Mean }=45.2 \\
\mathrm{SD}=10.9 \\
\text { Range }=18-68\end{array}$ & $\begin{array}{c}\text { Mean }=46.5 \\
S D=10.8\end{array}$ & $\begin{array}{c}\text { Mean }=44.4 \\
\mathrm{SD}=11\end{array}$ & $\begin{array}{c}t=1.64 \\
p=.2\end{array}$ \\
\hline Sex & $\begin{array}{l}M=45(24.6 \%) \\
F=138(75.4 \%)\end{array}$ & $\begin{array}{l}M=21(30 \%) \\
F=49(70 \%)\end{array}$ & $\begin{array}{l}\mathrm{M}=24(21.2 \%) \\
\mathrm{F}=89(78.8 \%)\end{array}$ & $\begin{aligned} x^{2} & =1.79 \\
p & =0.2\end{aligned}$ \\
\hline $\begin{array}{l}\text { Baseline weight } \\
\text { (kgs) }\end{array}$ & $\begin{array}{c}\text { Mean }=141.3 \\
\mathrm{SD}=28.0 \\
\text { Range }=96.5-250.8\end{array}$ & $\begin{array}{c}\text { Mean }=141.6 \\
S D=31.2\end{array}$ & $\begin{array}{c}\text { Mean }=141.2 \\
S D=25.9\end{array}$ & $\begin{array}{l}t=.009 \\
p=0.9\end{array}$ \\
\hline Baseline BMI & $\begin{array}{c}\text { Mean=50.2 } \\
S D=7.85\end{array}$ & $\begin{array}{c}\text { Mean }=49.2 \\
\mathrm{SD}=8.0\end{array}$ & $\begin{array}{c}\text { Mean }=50.9 \\
S D=7.7\end{array}$ & $\begin{array}{l}t=1.91 \\
P=0.2\end{array}$ \\
\hline Type of surgery & $\begin{array}{c}\text { Bypass }=170(92.9 \%) \\
\text { Band }=9(4.9 \%) \\
\text { Sleeve }=4(2.2 \%)\end{array}$ & $\begin{array}{c}\text { Bypass }=66(94.3 \%) \\
\text { Band }=4(5.7 \%)\end{array}$ & $\begin{array}{c}\text { Bypass }=104(92 \%) \\
\text { Band }=5(4.4 \%) \\
\text { Sleeve= } 4(3.5 \%)\end{array}$ & $\begin{aligned} x^{2} & =2.65 \\
p & =0.3\end{aligned}$ \\
\hline Ethnic group & $\begin{array}{c}\text { Black= } 2(1.1 \%) \\
\text { Asian= } 2(1.1 \%) \\
\text { White }=177(96.7 \%) \\
\text { Other= } 2(1.1 \%)\end{array}$ & $\begin{array}{c}\text { Black=1 }(1.4 \%) \\
\text { Asian= } 0 \\
\text { White }=68(97.1 \%) \\
\text { Other= } 1(1.4 \%)\end{array}$ & $\begin{array}{c}\text { Black }=1(0.9 \%) \\
\text { Asian= } 2(1.8 \%) \\
\text { White }=109(96.5 \%) \\
\text { Other= } 1(0.9 \%)\end{array}$ & $\begin{aligned} x^{2} & =1.48 \\
p & =0.7\end{aligned}$ \\
\hline $\begin{array}{l}\text { Education } \\
\text { <sec school } \\
\text { Sec school } \\
\text { Prof qual } \\
\text { Degree } \\
\text { High degree }\end{array}$ & $\begin{array}{c}5(2.7 \%) \\
78(42.9 \%) \\
70(38.5 \%) \\
25(13.7 \%) \\
4(2.2 \%)\end{array}$ & $\begin{array}{c}1(1.4 \%) \\
29(41.4 \%) \\
26(37.1 \%) \\
13(18.6 \%) \\
1(1.4 \%)\end{array}$ & $\begin{array}{c}4(3.6 \%) \\
49(43.8 \%) \\
44(39.3 \%) \\
12(10.7 \%) \\
3(2.7 \%)\end{array}$ & $\begin{aligned} x^{2} & =3.07 \\
p & =0.6\end{aligned}$ \\
\hline $\begin{array}{l}\text { Living Status } \\
\text { Alone } \\
\text { Co habiting }\end{array}$ & $\begin{array}{c}32(17.5 \%) \\
151(82.5 \%)\end{array}$ & $\begin{array}{l}12(17.1 \%) \\
58(82.9 \%)\end{array}$ & $\begin{array}{l}20(17.7 \%) \\
93(82.3 \%)\end{array}$ & $\begin{aligned} X^{2} & =.009 \\
p & =0.9\end{aligned}$ \\
\hline Final weight at 1 year $(\mathrm{kg})$ & $\begin{array}{c}\text { Mean }=94.8 \\
S D=20.0\end{array}$ & $\begin{array}{c}\text { Mean }=95.7 \\
S D=21.4\end{array}$ & $\begin{array}{c}\text { Mean }=94.1 \\
S D=18.9\end{array}$ & $\begin{array}{l}\mathrm{t}=.225 \\
\mathrm{p}=0.6\end{array}$ \\
\hline
\end{tabular}


Citation: Hollywood A, Ogden J, Pring C. The Impact of Psychological Support on Psychological Outcomes and Patients' Experiences of the Bariatric Service 1 and 2 Years after Bariatric Surgery. J Obes Bariatrics. 2015;2(1): 7.

of 16 patients took part in telephone interviews concerning their experiences of surgery.

\section{Intervention}

Patients allocated to the usual care (control) group received preoperative tests and a standard diet sheet postoperatively informing them about their desired diet and the stages of food progression from only consuming liquids to soft food then back to all foods. Patients returned for surgery approximately 2 weeks later, and after a median post-surgical stay of two nights, they were discharged home. They then returned to the clinic at 6 weeks, 3, 6 and 12 months to see the dietician and/or specialist nurse. Patients allocated to the BRS (intervention) group received usual care as described above plus three one-to-one 50 -min sessions with a health psychologist 2 weeks preoperatively, postoperatively (before they were discharged from hospital) and at 3 months follow-up. A one to one approach was chosen as a group would have been problematic to deliver with any consistency due to variability in appointment times and surgery dates. This would have meant that the composition of the groups could not have been kept consistent across the three delivery time points and participants could have ended up in groups with different patients. Furthermore, the intervention was tailored to address the individual needs of each patient. Again this would have been hard to achieve within a group.

The health psychologist used both didactic and non-didactic methods and addressed five key factors: (i) knowledge (i.e. information about dietary change), (ii) beliefs (concerning the causes and solutions to obesity), (iii) behaviours (with a focus on diet and physical activity), (iv) coping strategies (i.e. managing emotions without using food, identifying alternative and healthy methods of coping, managing other addictions) and (v) adjustment (i.e. exploring ways to work with the restriction imposed by the operation). Details concerning the structure of the sessions can be found in the study protocol [27].

\section{Measures}

i) Participant demographics: Weight, BMI, age, sex, ethnic group, education, living status and years trying to lose weight were assessed at baseline.

ii) Weight loss: patient weight was obtained in the clinic 2 weeks preoperatively and postoperatively at 12 months. The primary outcome was change in BMI by one year. These data have been published elsewhere [27].

iii) Psychological outcomes: Patients completed the following psychological measures in the clinic 2 weeks preoperatively (a paper questionnaire) and postoperatively at 12 months (mostly using an online survey): i) Mood (assessed in terms of depression, anxiety, vigour and fatigue, using the Profile of Mood States, [29]); Quality of life (assessed in terms of Activities of Daily Living (SF-36, [30]), Individualised Quality of Life (SEIQoL [31]) and the single item health status scale [32]; iii) Coping (approach coping style and behavioural disengagement were assessed using the COPE; [33]); Eating behaviour (emotional eating was assessed using the subscale from the DEBQ, [34] and changes in hunger, a desire to eat fatty foods, sweet foods or healthy foods using a measure of behaviour specific to changes post bariatric surgery [16]). These measures all show good levels of validity and have been used previously in the context of bariatric surgery (see Ogden and Hollywood for a review [35]).

iv) Patients' experiences of the surgical process: At 24 months patients' experiences of their operation and support with a focus on timing and content were collected using both written data and qualitative interviews: a) written data: this involved the completion of rating scales and open text boxes. Beliefs about timing were assessed using the following questions repeated for each time point: "If you could have some form of psychological support to help you to manage your weight loss surgery, to what extent do you think it should be: before /immediately after / 3 months after / 6 months after / 12 months after / 18 months after / 24 months after surgery", which were rated on 5 point Likert scales ranging from 'not at all (1) to 'totally' (5). Beliefs about content were assessed using the following questions: "To what extent did these support sessions: help to change my behaviour / help me manage my emotions /help me to find ways to cope with my surgery /help me to change my relationship with food" which were rated using 5 point Likert scales ranging from 'totally disagree' (1) to 'totally agree' (5). b) Qualitative data: This involved telephone interviews which were recorded and transcribed verbatim. Those in the intervention group were asked specific questions concerning the timing and content of the BRS. All patients were asked questions about optimal timing and content for psychological support such as "have you received any support with changing your behaviour or coming to terms with the effects of the surgery?"; 'was this support useful?'; 'how could this support have been improved?'

\section{Data analysis}

Patient demographics at baseline, for those with psychological outcomes data at 12 months were analysed using descriptive statistics. The impact of the BRS intervention on psychological outcomes at 12 months was assessed using ANOVA with time (baseline vs 12 months follow up) as the within subject factor and condition (usual care vs BRS intervention) as the between subjects factor. The patient experiences in terms of the rating scales were analysed using descriptive analysis. The open text boxes and qualitative interview data were analysed using content analysis.

\section{Results}

\section{Patient demographics}

Patient demographics for those with psychological outcome data by condition are shown in Table 2 .

The results showed that the majority were women (70\%), white (97\%), had a mean age of 46 years, cohabited with someone (83\%), had a mean baseline weight of $141.6 \mathrm{~kg}$, a mean baseline BMI of 49 and a final weight by 1 year of $95.7 \mathrm{~kg}$. The majority had been educated up to the end of secondary school (41\%) or had a professional qualification $(37 \%)$. Nearly all patients had received a gastric bypass $(n=66)$ and only 4 had received a gastric band $(n=4)$, The two groups were comparable in terms of baseline demographics and type of surgery received. Those in the intervention group, however, were significantly heavier in terms of both baseline weight and BMI compared to those in the usual care group. Baseline weight was therefore used as a covariate in all subsequent analysis. Patients $(n=16)$ who took part in 
Citation: Hollywood A, Ogden J, Pring C. The Impact of Psychological Support on Psychological Outcomes and Patients' Experiences of the Bariatric Service 1 and 2 Years after Bariatric Surgery. J Obes Bariatrics. 2015;2(1): 7.

ISSN: $2377-9284$

Table 2: Patient demographics for psychological outcomes $(n=70)$.

\begin{tabular}{|c|c|c|c|c|}
\hline & $\begin{array}{l}\text { All participants } \\
\qquad(n=70)\end{array}$ & Usual care $(n=32)$ & Intervention $(n=38)$ & $t / X^{2}$ \\
\hline Age (yrs) & $\begin{array}{c}\text { Mean }=46.5 \\
S D=0.77 \\
\text { Range }=21-68\end{array}$ & $\begin{array}{c}\text { Mean }=47.5 \\
S D=9.9\end{array}$ & $\begin{array}{c}\text { Mean }=45.7 \\
S D=11.5\end{array}$ & $\begin{array}{l}t=0.7 \\
p=0.5\end{array}$ \\
\hline Sex & $\begin{array}{l}M=21(30 \%) \\
F=49(70 \%)\end{array}$ & $\begin{array}{l}M=8(25 \%) \\
F=24(75 \%)\end{array}$ & $\begin{array}{l}M=13(34.2 \%) \\
F=25(65.8 \%)\end{array}$ & $\begin{array}{l}X^{2}=0.7 \\
p=0.4\end{array}$ \\
\hline Baseline weight (kgs) & $\begin{array}{c}\text { Mean }=141.57 \\
S D=31.23 \\
\text { Range }=99.8-250.8\end{array}$ & $\begin{array}{c}\text { Mean=131.9 } \\
S D=22.5\end{array}$ & $\begin{array}{c}\text { Mean }=149.7 \\
S D=35.3\end{array}$ & $\begin{array}{c}t=2.6 \\
p=0.01^{*}\end{array}$ \\
\hline Baseline BMI & $\begin{array}{c}\text { Mean }=49.21 \\
\mathrm{SD}=8.09\end{array}$ & $\begin{array}{c}\text { Mean }=46.6 \\
S D=6.3\end{array}$ & $\begin{array}{c}\text { Mean }=51.4 \\
\mathrm{SD}=8.7\end{array}$ & $\begin{array}{c}t=2.7 \\
p=0.008 *\end{array}$ \\
\hline Type of Surgery & $\begin{aligned} \text { Bypass } & =66(94.3 \%) \\
\text { Band } & =4(5.7 \%)\end{aligned}$ & $\begin{aligned} \text { Bypass } & =29(90.6 \%) \\
\text { Band } & =3(4.3 \%)\end{aligned}$ & $\begin{aligned} \text { Bypass } & =37(97.4 \%) \\
\text { Band } & =1(1.4 \%)\end{aligned}$ & $\begin{array}{c}\mathrm{X}^{2}=1.47 \\
\mathrm{p}=0.2\end{array}$ \\
\hline Ethnic group & $\begin{array}{c}\text { Black=1 }(1.4 \%) \\
\text { White=68 }(97.1 \%) \\
\text { Other=1 }(1.4 \%)\end{array}$ & $\begin{array}{c}\text { Black=1 }(3.1 \%) \\
\text { White= } 31(96.9 \%)\end{array}$ & $\begin{array}{c}\text { White=37 }(97.4 \%) \\
\text { Other=1 }(2.6 \%)\end{array}$ & $\begin{array}{c}X^{2}=2.03 \\
p=0.4\end{array}$ \\
\hline $\begin{array}{l}\text { Living status } \\
\text { Alone } \\
\text { Cohabiting }\end{array}$ & $\begin{array}{l}12(17.15) \\
58(82.9 \%)\end{array}$ & $\begin{array}{c}7(21.9 \%) \\
25(13.2 \%)\end{array}$ & $\begin{array}{c}5(78.1 \%) \\
33(86.8 \%)\end{array}$ & $\begin{array}{l}X^{2}=2.5 \\
p=0.6\end{array}$ \\
\hline Final weight at 1 year $(\mathrm{kg})$ & $\begin{array}{c}\text { Mean }=95.7 \\
S D=21.4\end{array}$ & $\begin{array}{c}\text { Mean= } 91.1 \\
S D=19.2\end{array}$ & $\begin{array}{c}\text { Mean }=99.4 \\
S D=22.7\end{array}$ & $\begin{array}{l}t=1.6 \\
p=0.1\end{array}$ \\
\hline
\end{tabular}

the qualitative component were representative of the psychological outcomes sample.

\section{Psychological outcomes}

The impact of the intervention compared to usual care on psychological outcomes from baseline pre surgery to 1 year follow up is shown in Table 3 .

On initial analysis the results showed significant effects of time for all variables (apart from approach coping and behavioural disengagement) indicating improvements for all patients regardless of condition on most psychological outcomes. In addition, the results showed significant time by condition interactions for depression, vigour, approach coping, individualised quality of life and hunger suggesting that improvements in these constructs were more pronounced in the intervention group. However, due to baseline differences in weight between the groups, baseline weight was entered as a covariate into all subsequent analyses. The results from this adjusted analysis showed no differences between the two conditions by 12 months follow up in terms of anxiety, fatigue, depression, behavioural disengagement coping, activities of daily living, health status, the desire to eat healthy foods, fatty foods or sweet foods or emotional eating. However, those patients who had received the BRS showed greater improvements in terms of vigour, individualised quality of life approach coping and hunger.

\section{Patients' experiences of support received for the surgical process}

Data from the rating scales, open text boxes and interviews at 24 months were analysed with a focus on patients' experiences concerning the actual and ideal timing along with the content of the support they received.

i) Actual timing: Of the patients in the intervention group who received additional support at baseline, immediately post-op and at 3 month follow up, most were satisfied with their experience with the majority $(\mathrm{n}=25 ; 92.6 \%)$ describing the timing as 'just at the right time' and only a small minority responding that the sessions were 'too early' ( $\mathrm{n}=9 ; 33.3 \%)$ or 'too late' $(\mathrm{n}=7 ; 25.9 \%)$. This was also reflected in the interview data with one patient reporting "time was very well spent, don't think I could have done it as well as I have without her help and understanding". However, whereas one-third said that there were 'too many sessions' $(n=9 ; 33.3 \%)$ a majority argued that there were 'too few sessions' $(\mathrm{n}=16 ; 59.2 \%)$.

ii) Ideal timing: All patients, regardless of condition, were also asked about the ideal timing for psychological support. The results showed that the majority of patients reported that they would prefer to receive psychological support at all suggested time points (before surgery $(n=56 ; 82.4 \%)$; immediate post operation $(n=53 ; 78 \%) ; 3$ months $(\mathrm{n}=55 ; 80.9 \%) ; 6$ months $(\mathrm{n}=54 ; 79.4 \%) ; 12$ months $(\mathrm{n}=53$, $78 \%) ; 18$ months $(\mathrm{n}=46 ; 67.7 \%)$ and 24 months $(\mathrm{n}=47 ; 69.2 \%))$. This majority, however, became gradually smaller the more distant the timing was from the date of the operation indicating that although the majority want support at all times, a larger majority would prefer it to be within the first rather than second year after their operation. This need for additional support was also illustrated in the interview data. For example, one patient reported "Further sessions would help me with my feelings regarding food"; one described how they were "worried that my relationship with food is going back to how it 
Citation: Hollywood A, Ogden J, Pring C. The Impact of Psychological Support on Psychological Outcomes and Patients' Experiences of the Bariatric Service 1 and 2 Years after Bariatric Surgery. J Obes Bariatrics. 2015;2(1): 7.

ISSN: $2377-9284$

Table 3: Impact of the BRS on psychological outcomes by one year (mean/SDs) (with baseline weight as a covariate).

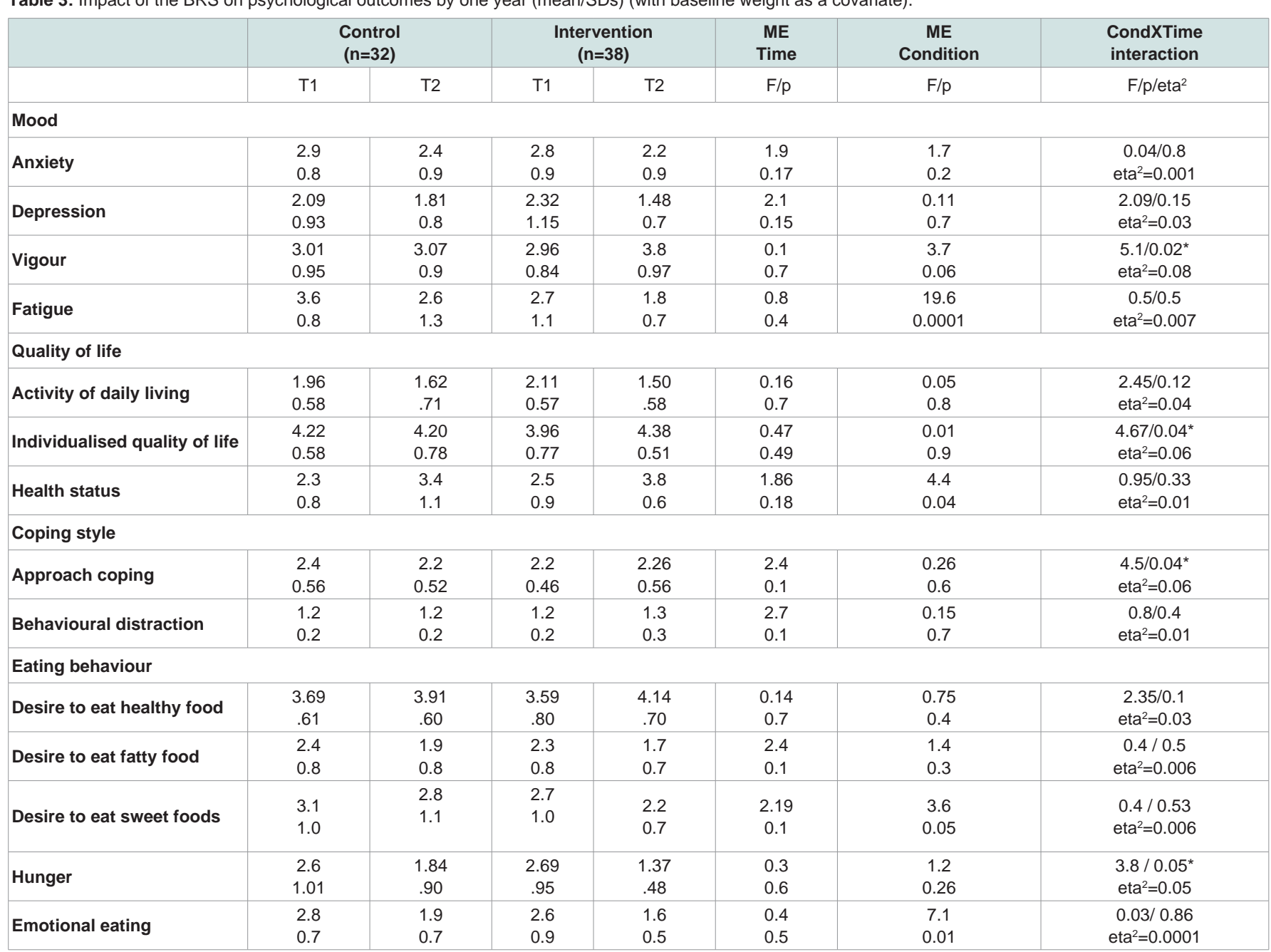

$\mathrm{ME}=$ main effect; ${ }^{\star} \mathrm{p}<0.05$

used to be" and another patient suggested "much better to have some emotional and practical support when restriction lifts and ability to eat eases!"

iii) Content: Patients' evaluation of the content of the additional psychological support from the BRS indicated that the large majority were positive about all aspects of the sessions they had received. In particular, the majority described the sessions as 'a good source of information' ( $\mathrm{n}=25 ; 92.6 \%)$; that they made them feel 'supported' $(\mathrm{n}=25 ; 92.6 \%)$ and 'listened to' $(\mathrm{n}=25 ; 92.6 \%)$ and that they helped them to 'change my behaviour' ( $\mathrm{n}=25 ; 92.6 \%)$; 'manage my emotions' $(\mathrm{n}=24 ; 68.9 \%)$; 'find ways to cope with my surgery' $(\mathrm{n}=23 ; 85.2 \%)$; 'change my relationship with food' ( $\mathrm{n}=22 ; 81.5 \%)$. This positive feedback was also reflected in the interviews. For example, one patient described how "Because my eating problems were emotionally based my time with her [the health psychologist] was invaluable". However, it was clear that the content of the sessions was more suited to some patients compared to others highlighting the need for some degree of patient selection. For example, when one patient was asked if they wanted more psychological support they responded "no...I didn't have a great deal of problems because I followed what I was told... .I followed it to the letter" whilst another was clear that "They [the health psychologist] didn't really change or affect my experience at all."

\section{Discussion}

The present study evaluated the impact of psychological support in the form of a health psychology led bariatric rehabilitation service and showed that although, as previously reported, the intervention had no impact upon weight loss by 1 year [27] some differences were found for psychological outcomes. In particular, after accounting for baseline weight, those who had received the additional psychological support pre, post and 3 months after surgery reported greater improvements in their levels of vigour, individualised quality of life, approach coping and hunger. This provides some support for previous studies arguing for the use of psychological support pre and post surgery $[17,18]$. It also offers a preliminary evidence base for the guidelines proposed by NICE [21] and AACE /TOS /ASMBS [22]. Accordingly, although no differences were found for weight loss, additional psychological support had some impact upon the patient's psychological state.

The present study also aimed to explore patients' experiences of the support they received with a particular focus on timing and 
Citation: Hollywood A, Ogden J, Pring C. The Impact of Psychological Support on Psychological Outcomes and Patients' Experiences of the Bariatric Service 1 and 2 Years after Bariatric Surgery. J Obes Bariatrics. 2015;2(1): 7.

content. Overall, the results from this analysis indicated that whilst the majority who received the BRS additional support were satisfied with this service in terms of timing and content and felt that it addressed their emotional and behavioural needs, many patients (regardless of condition) would have preferred support for a longer follow up period rather than just in the first three months. Furthermore, a minority felt that psychological support was unnecessary as they considered their problems not to be emotionally based and a third of those who had taken part in the BRS felt that 3 sessions was too many.

There are some problems with this study that need to be considered. First, although the study was fully powered for the preliminary analysis focusing on weight loss, dropout rates meant that complete psychological data was only obtained from under half of the initial sample. The results therefore need to be treated with caution not only in terms of the chance of making Type 2 errors but also as those patients who completed psychological measures may have shown a different profile of psychological change than those who did not. Second, although significant, the effects sizes were small for most outcomes. Third, the study explored patients experiences of support which relies upon their version of what they believe would benefit their health outcomes. Although such data provides a useful insight into what patients would like, it may not be the case that what they would like is necessarily what would be better for them. Finally, longer term follow up data is needed to assess whether changes in psychological state persist over time and whether these are linked to subsequent changes in weight.

To conclude, the results from the present study indicate that additional psychological support was related to some improvements in psychological outcomes by 1 year. Further, at 24 months although the majority of patients expressed satisfaction with this additional support in terms of timing and content, the majority of all patients would have liked more support over a longer period of time. This suggests that additional psychological support can be beneficial and is desired by patients. However, the small effects sizes and the dissenting minority of patients suggest that this conclusion does not stand for all patients. In particular, some patients showed no benefit and some found the support unnecessary. Further, many constructs showed no differential response to the additional psychological support. Accordingly, this study highlights variability in both the effectiveness of psychological support and the needs of bariatric patients and therefore emphasises the need for patient selection and targeting those patient who would most benefit. It is therefore argued, in light of the results presented in both the previous paper [27] and this current one, that there remains very little evidence for the effectiveness of psychological support for either weight loss or psychological outcomes after bariatric surgery. Therefore, if patients undergoing bariatric surgery are to be offered psychological support, further research is required, first, to identify when it can be delivered most effectively, and second, to develop an understanding of who would most benefit from this additional form of intervention.

\section{References}

1. Picot J, Jones J, Colquitt JL, Gospodarevskaya E, Loveman E, et al. (2009) The clinical effectiveness and cost-effectiveness of bariatric (weight loss) surgery for obesity: a systematic review and economic evaluation. Health Technol Assess 13: 1-190.

2. Sjostrom L, Nabro K, Sjostrom CD, Karason K, Larsson B, et al. (2007)
Effects of bariatric surgery on mortality in Swedish obese subjects. N Engl J Med 357: 741-752.

3. Nguyen NT, Silver M, Robinson M, Needleman B, Hartley G, et al. (2006) Result of a national audit of bariatric surgery performed at academic centers. a 2004 University Health System Consortium Benchmarking Project. Arch Surg 141: 445-449.

4. Courcoulas AP, Christian NJ, Belle SH, Berk PD, Flum DR, et al. (2013) Weight change and health outcomes at 3 years after bariatric surgery among individuals with severe obesity. JAMA 310: 2416-2425.

5. Karlsson J, Sjöström L, Sullivan M (1998) Swedish obese subjects (SOS)-an intervention study of obesity. Two-year follow-up of health related quality of life (HRQL) and eating behaviour after gastric surgery for severe obesity. Int J Obes Relat Metab Disord 22: 113-126.

6. Bocchieri LE, Meana M, Fisher BL (2002) A review of psychosocial outcomes of surgery for morbid obesity. J Psychosom Res 52: 155-165.

7. O'Brien PE, McPhail T, Chaston TB, Dixon JB (2006) Systematic review of medium-term weight loss after bariatric operations. Obes Surg 16: 10321040 .

8. Buchwald H, Avidor Y, Braunwald E, Jensen MD, Pories W, et al. (2004) Bariatric surgery: a systematic review and meta-analysis. JAMA 292: 17241737.

9. Magro DO, Geloneze B, Delfini R, Paraja BC, Callejas F, et al. (2008) Longterm weight regain after gastric bypass: a 5-year prospective study. Obes Surg 18: 648-651.

10. Odom J, Zalesin KC, Washington TL, Miller WW, Hakmeh B, et al. (2010) Behavioral predictors of weight regain after bariatric surgery. Obes Surg 20 : 349-356.

11. Semanscin-Doerr DA, Windover A, Ashton K, Heinberg LJ (2010) Mood disorders in laparoscopic sleeve gastrectomy patients: does it affect early weight loss? Surg Obes Relat Dis 6: 191-196.

12. Saltzman E, Anderson W, Apovian CM, Boulton H, Chamberlain A, et al. (2005) Criteria for patient selection and multidisciplinary evaluation and treatment of the weight loss surgery patient. Obes Res 13: 234-243.

13. Wood KV, Ogden J (2012) Explaining the role of binge eating behaviour in weight loss post bariatric surgery. Appetite 59: 177-180.

14. Ogden J, Clementi C, Aylwin S (2006) The impact of obesity surgery and the paradox of control: A qualitative study. Psychol Health 21: 273-293.

15. Ogden J, Avenell S, Ellis G (2011) Negotiating control: patients' experiences of unsuccessful weight-loss surgery. Psychol Health 26: 949-964.

16. Ogden J, Clementi C, Aylwin S, Patel A (2005) Exploring the impact of obesity surgery on patient's health status: a quantitative and qualitative study. Obes Surg 15: 266-272.

17. Kalarchian MA, Marcus MD, Courcoulas AP, Cheng Y, Levine MD (2013) Preoperative lifestyle intervention in bariatric surgery: initial results from a randomized, controlled trial. Obesity (Silver Spring) 21: 254-260.

18. Kalarchian MA, Marcus MD, Courcoulas AP, Cheng $Y$, Levine MD, et al. (2012) Optimizing long-term weight control after bariatric surgery: a pilot study. Surg Obes Relat Dis 8: 710-715.

19. Heinberg LJ (2012) Comment on: Optimizing long-term weight control after bariatric surgery: a pilot study. Surg Obes Relat Dis 8: 715-716.

20. Henrickson HC, Ashton KR, Windover AK, Heinberg LJ (2009) Psychological considerations for bariatric surgery among older adults. Obes Surg 19: 211216.

21. National Institute for Health and Care Excellence. Specifying a bariatric surgical service for the treatment of people with severe obesity.

22. Mechanick JI, Youdim A, Jones DB, Garvey WT, Hurley DL, et al (2013) Clinical practice guidelines for the perioperative nutritional, metabolic, and nonsurgical support of the bariatric surgery patient-2013 update: cosponsored by American Association of Clinical Endocrinologists, The Obesity Society, 
Citation: Hollywood A, Ogden J, Pring C. The Impact of Psychological Support on Psychological Outcomes and Patients' Experiences of the Bariatric Service 1 and 2 Years after Bariatric Surgery. J Obes Bariatrics. 2015;2(1): 7.

and American Society for Metabolic \& Bariatric Surgery. Obesity (Silver Spring) 21 Suppl 1: S1-S27.

23. Ades PA (2001) Cardiac rehabilitation and secondary prevention of coronary heart disease. N Engl J Med 345: 892-902.

24. Petrie KJ, Cameron LD, Ellis CJ, Buick D, Weinman J (2002) Changing illness perceptions after myocardial infarction: an early intervention randomized controlled trial. Psychosom Med 64: 580-586.

25. Egbert LD, Battit GE, Welch CE, Bartlett MK (1964) Reduction of postoperative pain by encouragement and instruction of patients. N Engl J Med 270: 825-

26. Johnston M, Vogele C (1993) Benefits of psychological preparation for surgery: a meta-analysis. Ann Behav Med 15: 245-256.

27. Ogden J, Hollywood A, Pring C (2015) The impact of psychological support on weight loss post weight loss surgery: a randomised control trial. Obes Surg 25: $500-505$

28. Hollywood A, Ogden J, Pring C (2012) The impact of a bariatric rehabilitation service on weight loss and psychological adjustment-study protocol. BMC Public Health 12: 275.
29. McNair D, Lorr M, Droppleman L (1992) Profile of Mood States (POMS). Educational and Industrial Testing Service.

30. Ware JE, Sherbourne CD (1992) The MOS 36-item short-form health survey (SF-36). I. Conceptual framework and item selection. Med Care 30: 473-483.

31. Hickey AM, Bury G, O'Boyle CA, Bradley F, O'Kelly FD, et al. (1996) A new short form individual quality of life measure (SEIQoL-DW): application in a cohort of individuals with HIVIAIDS. BMJ 313: 29-33.

32. Idler EL, Angel RJ (1990) Self-rated health and mortality in the NHANES-I Epidemiologic Follow-up Study. Am J Public Health 80: 446-452.

33. Carver CS, Scheier MF, Weintraub JK (1989) Assessing coping strategies: a theoretically based approach. J Pers Soc Psychol 56: 267-283.

34. Van Strien T, Frijters JE, Bergers GP, Defares PB (1986) The dutch eating behaviour questionnaire (DEBQ) for assessment of restrained, emotional and external eating behaviour. Int J Eat Disord 5: 295-315.

35. Ogden J, Hollywood A. Health related quality of life before and after bariatric surgery. In: Obesity and Bariatric Surgery: A Practical Guide. ed. Sanjay Agrawal. Springer-Verlag. In press.

\section{Acknowledgements}

The authors acknowledge the support of the Research for Patient Benefit programme as part of the National Institute for Health Research through the Comprehensive Clinical Research Network and the Western Sussex Hospitals NHS Trust. They would also like to thank Dr Kerry Wood for carrying out the interviews and all the staff and patients at St Richards Bariatric Service for facilitating and engaging with this research.

Funding: This study was funded by RfPB NIHR. Grant number: PB-PG-0909-20178. This trial was conducted and data collected with complete independence of the researchers from the RfPB NIHR.

Trial registration: ClinicalTrials.gov NCT01264120. 